Here $\sum(x)$ comes from the classical vortex solution in the $A_{0}=0$ gauge. ${ }^{4}$

Finally, I wish to remark that we have done a matching between two different perturbations schemes; namely, between the standard perturbation and the one around the instanton. This is a new concept in field theory which may have other applications besides tunneling as Gribov's problem. ${ }^{3}$

\section{References}

1. J. L. Gervais and B. Sakita: CCNY reportHEP/76-11, 1976; Phys. Rev. D16 (1977) 3507.
2. H. J. de Vega, J. L. Gervais and B. Sakita: Nucl. Phys. B139 (1978) 20; Paris preprint LPTHE 78/08 to appear in Nucl. Phys. B.

3. H. L. Gervais and B. Sakita: CCNY reportHEP/78-5 to appear in Phys. Rev. D.

4. H. J. de Vega, J. L. Bervais and B. Sakita: Paris preprint LPTHE 78-09 to appears in Phys. Rev. D.

5. For a review on the subjects of refs. 1-4 see, J. L. Gervais: Les Houches Winter School, March 1978. Paris preprint LPTENS 78/19.

6. C. N. Yang: talk given in Session C2, this Conference.

7. C. Callan: talk given in Session $\mathrm{C} 1$, this Conference.

Proc. 19th Int. Conf. High Energy Physics

ToKyo, 1978

\title{
C 3 Resonance Properties in Quantum Chromodynamics
}

\author{
Presented by V. I. ZaKHAROV
}

ITEP, Moscow

The purpose of this Talk is to provide a short guide to a rather extensive work preprinted in ITEP this year. ${ }^{1}$

The outcome of the work is the QCD sum rules which are sensitive to a single resonance, say, $\rho$-meson. An example of the result obtained which is easy to memorize is

$$
{ }_{4 \pi}^{2} \simeq \begin{gathered}
2 \pi \\
e
\end{gathered}
$$

where $e$ is the base of natural logs and the Ihs is essentially the $\rho$-meson electronic width $\Gamma\left(\rho \rightarrow \mathrm{e}^{+} \mathrm{e}^{-}\right)=\alpha^{2} / 3 \cdot m_{\rho} \cdot\left(4 \pi / g_{\rho}^{2}\right) . \quad$ Equation (1) is not mnemonic but serious (to say nothing that it is in excellent agreement with the data).

There is a long way before we arrive at (1) (as well as many sideways). I can only touch upon the main points and present them in the following order. a) basic idea, b) and c) are principal technical devices and d) applications.

a) Let me recall you first the QCD classics on $\mathrm{e}^{+} \mathrm{e}^{-}$annihilation. The prediction is that at high energy the experimental cross section $\sigma_{\text {phys }}$ coincides with the quark one, $\sigma_{\text {quark }}$, which is calculable via simple quark graphs.

A bit more precise working is to say that the following sum rule holds

$$
\int \frac{\sigma_{\mathrm{phys}}(s) \mathrm{d} s}{\left(s+Q^{2}\right)} \simeq \int \frac{\sigma_{\mathrm{quark}}(s) \mathrm{d} s}{\left(s+Q^{2}\right)}
$$

provided $|Q|^{2}$ is large. The standard numerical prediction corresponds to keeping graphs $1 \mathrm{a}, \mathrm{b}$ for the photon-induced polarization operator.

Now, if $Q^{2}$ is decreased, physical cross section becomes structured due to the resonances and the central question is what kind of theoretical contribution is responsible for this. Our guess is that low momentum quark and gluon lines, $p^{2}, k^{2} \leq \mu^{2}, \mu$ being some mass scale, must be treated more carefully.

Thus, Fig. $1 \mathrm{~b}$ is transformed into the graphs of Fig. 2 where the external lines are annihilated by vacuum. This annihilation is accounted for phenomenologically, and is due to the nonperturbative effects. The route of large $Q^{2}$ flow is indicated by arrows. The propagation of the corresponding particles is described by free field theory due to the asymptotic freedom

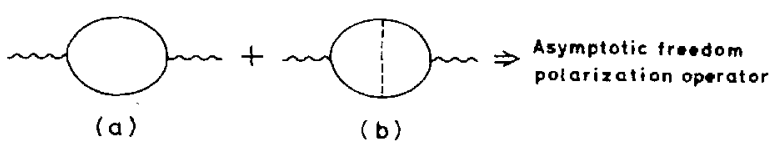

Fig. 1. 


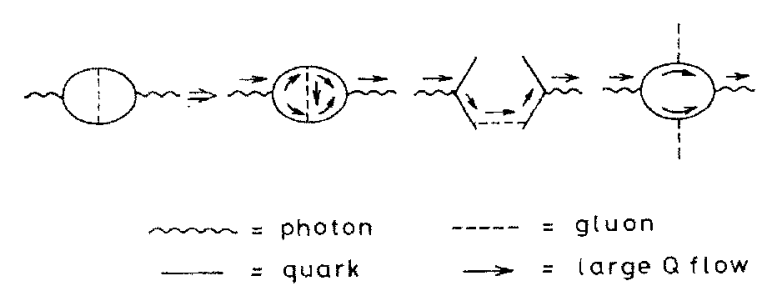

Fig. 2 ,

of QCD.

b) The central technical point is that there exists a well defined procedure to realize the program. Namely, one can rely on the standard operator expansion (O.E.), to treat the first power corrections in $Q^{-2}$.

$$
\int \mathrm{e}^{i q x} T\left\{j_{\mu}(x) j_{\nu}(0)\right\} \mathrm{d}^{4} x=\left(q_{\mu} q_{\nu}-g_{\mu \nu} q^{2}\right) \sum c_{n} O_{n}
$$

the few lower dimension operators being $O_{n}=I, G_{\mu \nu}^{a} G_{\mu \nu}^{a}, m \psi \bar{\phi}, \bar{\zeta} \Gamma \phi \bar{\delta} \Gamma \psi$ where $G_{\mu \nu}^{a}$ is the gluon field strength, $\phi$ is a quark field and $\Gamma$ are some matrices.

O.E. is so widely used nowadays that it might look strange to dwell upon it here. But in fact all the discussion so far refers to the perturbation theory where one has:

1) O.E. is OK.

2) Taking vacuum average of $\sum_{n} c_{n} O_{n}$ picks up the unity operator.

We are interested in nonperturbative effects of QCD, however, and both statements are modified:

1') O.E. is meaningful only as far as the corresponding matrix elements are dominated by the large scale, i.e., $Q^{2}$ independent fluctuations.

$\left.2^{\prime}\right)$ Apart from the unity operator the others develop nonvanishing vacuum expectation as well $\left(\left\langle 0\left|G_{\mu \nu}^{a} G_{\nu \mu}^{a}\right| 0\right\rangle,\langle 0|\bar{\phi} \phi| 0\rangle \cdots \neq 0\right)$.

Fortunately enough, we do know that at short distances the leading nonperturbative effects are given by instantons and are able to justify the use of operator expansion for up to quite a high power of $\left(Q^{-2}\right)$.

c) In fact we use a new form of sum rules which comes out by applying some operator $\hat{L}$ both to the dispersion and QCD representations for the polarization operators. The result looks as

$$
\begin{gathered}
\int \mathrm{e}^{-S ! M 2}\left[\sigma_{\mathrm{phys}}(s)-\sigma_{\mathrm{quark}}(s)\right] \mathrm{d} s \\
=\frac{h_{2}}{M^{4}}+\frac{h_{3}}{M^{6}}+0\left(M^{-8}\right),
\end{gathered}
$$

where the coefficients $h_{2,3}$ are given in terms of $\left\langle 0\left|G^{2}\right| 0\right\rangle,\left\langle 0\left|\phi^{4}\right| 0\right\rangle$. Note the appearance of a sharp exponential weight in the integrals over the cross sections. The precise form of operator $\hat{L}$ is

$$
\begin{aligned}
& \hat{L}=\lim _{n \rightarrow \infty} \frac{1}{(n-1) !}\left(Q^{2} \mathrm{~d} Q^{2}\right)^{n}, \\
& \text { with } \frac{Q^{2}}{n}=M^{2}, Q^{2}, n \rightarrow \infty .
\end{aligned}
$$

It is amusing to find that applying the operator $\hat{L}$ is equivalent to the Borel summation of the power corrections. Thus we suppress higher powers in $Q^{-2}$ which are neglected. The procedure can be reiterated but the second Borel transform would introduce a sign alternating weight function. Therefore the first Borel transform is the best one to study resonances.

d) Applications of the sum rules are quite numerous since we can try different currents $J_{\mu}$. Our claim is that power corrections become essential only at a rather low mass $M^{2} \simeq m_{\rho}^{2}$ so that integral over $\sigma_{\text {phys }}(s)$ is saturated by a single resonance. Once we get power corrections of order $0.1-0.2$ of the integral over the quark cross section we stop the calculation as not to go into the mass of higher order terms in $\mathrm{M}^{-2}$.

In fact, for a well defined reason terms $\sim M^{-4}, M^{-6}$ become comparable at this point while higher powers of $M^{-2}$ are still hopefully much smaller.

Thus, quantitative results require two parameters $\left\langle 0\left|G_{\mu_{\nu}}^{a} G_{\mu_{\nu}}^{a}\right| 0\right\rangle,\langle 0|\bar{\phi} \Gamma \phi \bar{\psi} \Gamma \phi| 0\rangle$. The latter one can be in fact borrowed from the studies of the chiral symmetry breaking so that there is no problem. As for the former one we see no way but to sacrifice one of the sum rules (for charmonium decays) to fix it from the data:

$$
\left\langle 0\left|{ }_{\pi}^{\alpha_{s}} G_{\mu_{\nu}}^{a} G_{\mu_{\nu}}^{a}\right| 0\right\rangle \simeq 0.012 \mathrm{GeV}^{4}
$$

Then we are free to evaluate the masses, mixing angles and leptonic widths of $\rho, \omega, \phi$. The axial vector channel (the $\pi-A_{1}$ system) is treated in the same way. Changing the current modifies only the coefficients $h_{2,3}$ in a well defined way and it is a real amusement to see that the resonance properties do comply with the change in the sum rules. 
The analysis seems to have some implications for the other models. In particular, via the triangle anomaly in the trace of the energymomentum tensor $\left\langle 0\left|\alpha_{s} G^{2}\right| 0\right\rangle$ is related to the vacuum energy due to the nonperturbative effects. This is the energy $\varepsilon$ outside the bag. Quarks most probably destoy vacuum fluctuations and, therefore, we propose an estimate for the constant $B$ of the bag model:

$$
\begin{aligned}
B & =-\varepsilon=-{ }_{32}^{9}\left\langle 0\left|{ }_{\pi}^{\alpha_{s}} G_{\mu^{\prime}}^{a} G_{\mu^{\prime \nu}}^{a}\right| 0\right\rangle \\
& =-0.0035 \mathrm{GeV}^{2}
\end{aligned}
$$

which is not bad numerically. Further argument can be found in the paper by Shuryak. ${ }^{2}$

\section{References}

1. M. Shifman, A. Vainshtein and V. Zakharov: preprints ITEP-73, 80, 81, 94, 99 (1978).

2. E. V. Shuryak and Novosibirsk: preprint 7854, May 1978. 



\title{
Session C5: Formal Theory
}

\author{
Chairman: A. MARTIN \\ Organizer: K. NishIJIMA \\ Scientific Secretaries: M. KoBAyashI \\ H. NAKAJIMA
}

1. Manifestly Covariant Canonical Formalism of Non-Abelian Gauge Theory and Quantum Gravity

N. NAKANISHI

2. Relativistic Nonlinear Quantum Mechanics

T. W. B. KIBBLE

3. Asymptotic Freedom and the Symplectic and $G_{2}$ Groups

M. Chatchian

(Friday, August 25, 1978; $9: 00-10: 20$ ) 